\title{
Patterns of oxygen saturation in prolonged recovery from COVID-19 pneumonia
}

F Asmodien, ${ }^{1}$ BSc (Physiotherapy); G Calligaro, ${ }^{2}$ BSc Hons, MB BCh, Dip PEC (SA), MMed, FCP; A Huisamen, ${ }^{1}$ BSc (Physiotherapy); N Kwinana, ${ }^{1}$ BSc (Physiotherapy); N Teixeira, ${ }^{1}$ MB ChB; T Ward, ${ }^{1}$ BSc (Physiotherapy); $\mathbf{S}$ J Reid, ${ }^{3}$ BSc (Med), MB ChB, MFamMed, PhD

${ }^{1}$ Cape Town, South Africa

${ }^{2}$ Department of Medicine, Faculty of Health Sciences, University of Cape Town, South Africa

${ }^{3}$ Primary Health Care Directorate, Faculty of Health Sciences, University of Cape Town, South Africa

Corresponding author: SJ Reid (steve.reid@uct.ac.za)

During the second wave of the COVID-19 pandemic in South Africa, a recurrent pattern of prolonged recovery after acute COVID-19 pneumonia, characterised by low oxygen saturation levels for $>2$ weeks, was observed in an intermediate-care facility in Cape Town. A case study together with a series of 12 patients is presented to illustrate this phenomenon, and two types of 'sats gap' are described, which were used by physiotherapists and doctors to monitor daily progress. We attempt to explain this prolonged recovery in terms of the possible pathophysiology, and suggest a number of learning points to guide further research.

S Afr Med J 2021;111(9):849-851. https://doi.org/10.7196/SAMJ.2021.v111i9.15722

In the context of intermediate care for COVID-19 patients de-escalated from acute-level hospitals, the doctors and physiotherapists working in the Mitchells Plain Hospital of Hope intermediate-care facility identified a recurrent pattern of recovery among a certain cohort of patients. We have observed that these patients show persistent dependence on low levels of inspired oxygen, with a significant gap in their oxygen saturation on inspired oxygen compared with room air. This is accompanied by a slow functional recovery that is facilitated by physiotherapy. For the purposes of presenting this series, we defined this prolonged recovery as 'functional dependence on $<5 \mathrm{~L}$ / min oxygen for $>2$ weeks after the acute phase of COVID pneumonia'. Intermediate care is defined as 'inpatient transitional care enabling patients to regain skills and abilities in daily living, involving postacute, rehabilitative and end-of-life care. ${ }^{[1]}$

\section{Case study}

We noted a numerical gap in oxygen saturation, which we have called the 'sats gap', between different levels of inspired oxygen. This is initially best illustrated through a single case study.

A 73-year-old woman (patient C in Table 1), with reasonably controlled type 2 diabetes, hypertension and chronic kidney disease, was admitted to a district hospital with COVID pneumonia, requiring $15 \mathrm{~L} / \mathrm{min}$ oxygen through a non-rebreather mask for 20 days. Thereafter she was transferred to the Mitchells Plain Hospital of Hope on nasal prongs oxygen, where she required $<5 \mathrm{~L} / \mathrm{min}$ oxygen for a further 33 days. She had no history of smoking, and was fully mobile prior to admission. In addition to her diabetes and hypertensive medications and routine enoxaparin sodium, she was given $40 \mathrm{mg}$ prednisone daily for 4 weeks, followed by a short course of $60 \mathrm{mg}$ daily for 5 days. She was discharged home with an oxygen saturation of $90 \%$ on room air, without home oxygen.

The graph of the patient's oxygen saturation readings in the first 2 weeks of her admission to intermediate care (Fig. 1) clearly shows the 'sats gap 1' between room air and low-flow oxygen, as well as the 'sats gap 2' between exertion and low-flow oxygen.

\section{Case series}

We present a series of 12 patients who conformed to the above definition (Table 1). They are ranked according to the total length of stay in hospital.

The average age of the patients was 62 years, with 8 out of the 12 being female. One was an ex-smoker, 8 had hypertension, and half had diabetes. The longest total hospital stay was 69 days, 42 at intermediate-care level. The recovery period was on average longer than the acute stage of illness ( 24 v. 18 days, respectively). Five of the 12 had had high-flow nasal oxygen (HFNO). Two were discharged on home oxygen and the rest on room air, with 5 of the 12 having saturation levels $<90 \%$. All received steroids for various lengths of time, with 3 being given lengthy prescriptions on discharge.

We observed that the following patterns related to recovery occurred commonly:

- The sats gap between low-flow oxygen and room air often remained static for an extended period of time, sometimes several weeks, after the acute stage was over (i.e. after week 2).

- The sats gap between room air at rest and room air after exertion often showed the most variability, and seemed to be a sensitive indicator of recovery.

- The length of time that the patient required post-exertion for their sats to return to baseline on room air gave us a more sensitive indicator of recovery. It was often the only indicator that showed any change from day to day, while the sats gaps remained static.

- Indicators of the functional capacity of the patient, e.g. the distance that they were able to walk off oxygen, the activities they were able to do, and the length of time that they felt comfortable off oxygen, were slightly more subjective but also useful signs of recovery. The ' 40 step test ${ }^{[2,3]}$ was introduced as a means of standardising the level of exertion for the purposes of comparison, even though it has not been validated.

- After 2 weeks of static indicators, having excluded other causes, we accepted a new baseline of oxygen saturation, even $<90 \%$, and sent the patient home. 


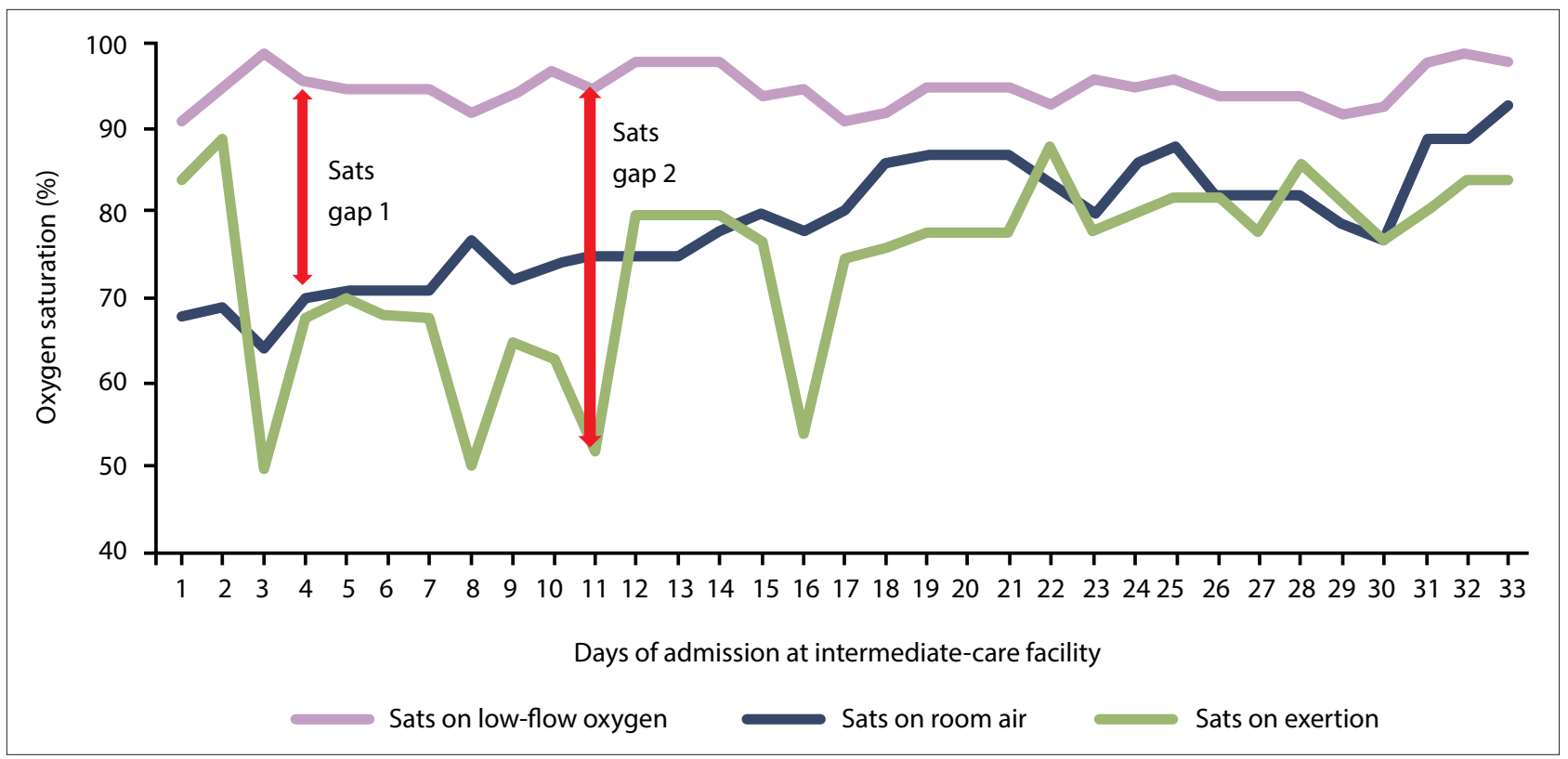

Fig. 1. Patient C's line graph, showing saturation levels throughout her admission at the intermediate-care facility.

- We often noted a persistent tachycardia ${ }^{[4]}$ of $>100 /$ min for which there was no obvious cause other than recovery from the pneumonia.

Other issues related to prolonged recovery that we observed were:

- The psychological state and degree of positive motivation of the patient was crucial to recovery, and this requires a whole-team approach. Individualised counselling can make a big difference, but the persistent encouragement of the patient by each team member boosted the recovery. Maintaining a high respiratory effort continuously for weeks requires a high degree of mental as well as physical stamina ('the marathon you never trained for').

- Close and accurate monitoring of the above indicators daily, and up to twice daily, is needed, in view of sudden deteriorations that are common and occasionally catastrophic.

- The diagnostic challenge is to differentiate an 'organising pneumonia' from other potential causes of slow recovery such as a pulmonary thrombus, a secondary bacterial lung infection, a pneumothorax or other common hidden causes such as congestive cardiac failure, Pneumocystis carinii pneumonia in HIV-positive patients, or reactivation of pulmonary tuberculosis. ${ }^{[5]}$

- A therapeutic challenge is related to the role of longer-term steroids $s^{[6]}$ in the absence of evidence for this scenario, as well as the need to cover with antibiotics in the absence of specific indications.

- The rehabilitation dilemma is how far to 'push' the patient each day, and exactly when to discharge from hospital, which is an incremental and individualised clinical decision that is largely managed by the physiotherapists.

\section{Discussion}

In seeking to explain this prolonged 'sats gap' phenomenon, we wonder whether the pneumonia affected the majority of both lungs in these patients, significantly reducing their capacity for oxygen transfer. This pulmonary dead space may relate to ground-glass opacities $^{[7]}$ seen on computed tomography (CT) scans. During recovery, there may be small parts of the lungs spared by the pneumonia that compensate effectively for the affected parts when small amounts of oxygen are given. The fact that $42 \%$ of our series of patients had been on HFNO or intubated in the intensive care unit would suggest that those whose lungs were most extensively affected were most likely to take longer than normal to recover. In the cases of static indicators of recovery leading to the conclusion of a reduced baseline (so-called 'permissive', 'silent' or 'happy' hypoxaemia ${ }^{[8]}$ ), the COVID pneumonia is likely to have permanently reduced oxygen transfer capacity as a result of destruction of alveolar architecture. Tobin et al. ${ }^{[9]}$ give an eloquent explanation of the various reasons for 'silent hypoxaemia' seen in the early stages of the pneumonia, including the way the respiratory centres respond to low levels of oxygen, the way the $\mathrm{CO}_{2}$ tension blunts the brain's response to hypoxaemia, and the inaccuracy of pulse oximetry. We accepted lower baseline oxygen saturation in patients who had been hypoxic for a protracted period, as they had effectively undergone the physiological process of acclimatisation similar to the response to high altitudes. For our patient $\mathrm{C}$, we eventually accepted a new lower baseline saturation level of $90 \%$, and in retrospect we could have discharged her a week earlier.

The diagnosis of an 'organising pneumonia' required the exclusion of other causes of persistent oxygen requirement, and a response to an escalation in steroid dose, in the setting of a suggestive CT scan. Without ready access to radiography, we were unable to make this diagnosis confidently, and gave short courses of higher-dose steroids to try to hasten recovery, with a few positive responses. Frija-Masson et al.$^{[10]}$ noted the poor correlation between the extent of pneumonia on CT and pulmonary function test (PFT) findings, with patients presenting abnormal results despite mild disease on CT. They recommended systematic functional assessment of SARS-CoV-2 patients with initial respiratory symptoms and longterm follow-up with high-resolution CT and PFTs. Alterations in ventilation/perfusion ratios are likely to be the major contributor to hypoxaemia. Increased dead space and therefore inefficient ventilation, particularly in the setting of low functional residual capacity (pulmonary restriction), and at times of increased respiratory rate such as during exertion, will cause rapid desaturation. This may explain the significant sats gap after exertion in our patients.

We offer these observations of prolonged recovery from COVID19 pneumonia in the spirit of curiosity, hoping that others observing extended recoveries of patients in similar situations at the intermediate level of care can help to build a more comprehensive picture of this phenomenon. 


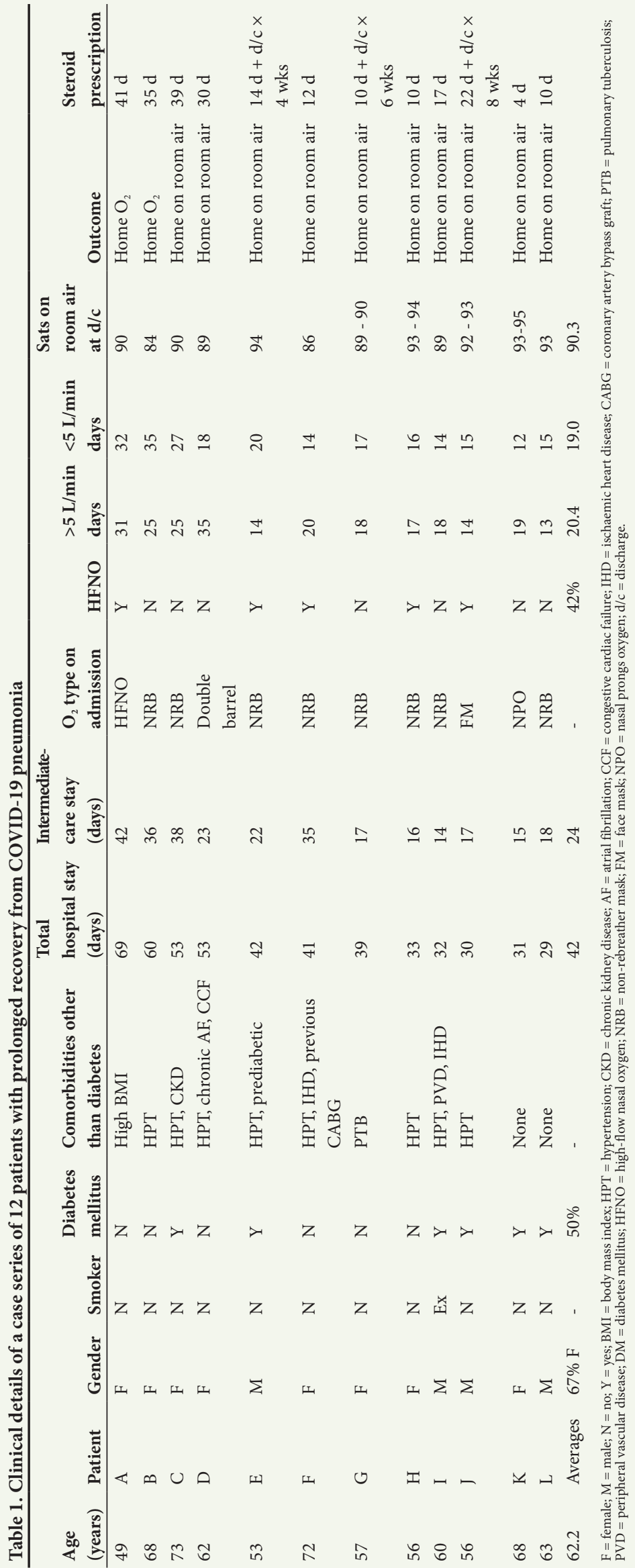

\section{Teaching points}

- Recovery from COVID-19 pneumonia varies widely depending on the severity of the acute stage.

- The prolonged dependence on low levels of inspired supplemental oxygen can be systematically monitored.

- The 'sats gaps' between the oxygen saturation levels on low-flow oxygen and room air, and post exertion, are useful monitoring measures.

- The length of time taken to return to a baseline oxygen saturation level after a standardised exertion is the most sensitive indicator of recovery.

- After severe COVID-19 pneumonia, many patients established a lower permanent baseline saturation level of $<90 \%$ which they tolerated well, so-called 'silent' hypoxaemia.

\section{Declaration. None.}

Ethical approval. This study was approved by the University of Cape Town Human Research Ethics Committee (ref. no. 502/2020).

Acknowledgements. The authors acknowledge the role of all the staff of the Mitchells Plain Hospital of Hope, and particularly Dr Elma de Vries for her leadership through the second phase of the pandemic.

Author contributions. All authors contributed to the development of the concept, the gathering of data and the drafting of the manuscript.

Funding. None.

Conflicts of interest. None.

1. Western Cape Government: Health. Healthcare 2030: The road to wellness. 2014 https://www.westerncape.gov.za/assets/departments/health/healthcare2030.pd (accessed 15 March 2021)

2. Kalin A, Javid B, Knight M, Inada-Kim M, Greenhalgh T. Direct and indirect evidence of efficacy and safety of rapid exercise tests for exertional desaturation in Covid-19: A rapid systematic review. Syst Rev 2021;10:77. https://doi org/10.1186/s13643-021-01620-w

3. NHS. Reference guide for Emergency Medicine: Specialty guides for patient management during the coronavirus pandemic. November 2020, revised May 2021. https://www.nice.org.uk/media/default/about/covid-19/specialty-guides/ management-emergency-department-patients.pdf (accessed 5 June 2021).

4. Cho JH, Namazi A, Shelton R, et al. Cardiac arrhythmias in hospitalized patients with COVID-19: A prospective observational study in the western United States. PLoS ONE 2020:15(12) e0244533. https://doiorg/10.1371/ journal pone 0244533

5. Kory P, Kanne JP. SARS-CoV-2 organising pneumonia: 'Has there been a widespread failure to identify and treat this prevalent condition in COVID-19?' BMJ Open Respir Res 2020;7(1):e000724. https://doi.org/10.1136 bmjresp-2020-000724

6. Mishra GP, Mulani J. Corticosteroids for COVID-19: The search for an optimum duration of therapy. Lancet Respir Med 2020;9(1):e8. https://doi.org/10.1016/ S2213-2600(20)30530-0

7. Wang $\mathrm{Y}$. Dong $\mathrm{C}, \mathrm{Hu} \mathrm{Y}$, et al. Temporal changes of $\mathrm{CT}$ findings in 90 patients with COVID-19 pneumonia: A longitudinal study. Radiology 2020;296:E55-E64. https://doi.org/10.1148/radiol.2020200843

8. Dhont S, Derom E, van Braeckel E, et al. The pathophysiology of 'happy' hypoxemia in COVID-19. Respir Res 2020;21:198. https://doi.org/10.1186/ s12931-020-01462-5

9. Tobin MJ, Laghi F, Jubran A. Why COVID-19 silent hypoxemia is baffling to physicians. Am J Respir Crit Care Med 2020;202(3):356-360. https://doi. org/10.1164/rccm.202006-2157CP

10. Frija-Masson J, Debray MP, Gilbert M, et al. Functional characteristics of patients with SARS-CoV-2 pneumonia at 30 days post-infection. Eur Respir 2020;56(2):2001754. https://doi.org/10.1183/13993003.01754-2020 